\title{
Online Channel Operation Mode: Game Theoretical Analysis from the Supply Chain Power Structures
}

\author{
Song Huang, Guocan Chen, Yanan Ma* \\ College of Economics \& Management, South China Agricultural University (China) \\ buangsong@scau.edu.cn, gcchen93@163.com,*Corresponding author: mavanan@scau.edu.cn
}

Received: July 2015

Accepted: November 2015

\section{Abstract:}

Purpose: Dual-channels have been widely used in practice, and the pricing decisions and the online channel operation mode choice have been the core problems in dual-channel supply chain management. This paper focuses on the online channel operation mode choice from the supply chain power structures based on game theoretical analysis.

Design/methodology/approach: This paper utilizes three kinds of game theoretical models to analyze the impact of supply chain power structures on the optimal pricing and online channel operation mode choice.

Findings: Results derived in this paper indicate that when the self-price elasticity is large, the power structures have no direct impact on the decisions. However, when the self-price elasticity is small and customers' preference for the online channel is low, then in the MS market, it is better for the retailer to operate the online channel, while in the RS market or in the VN market, it is better for the manufacturer to operate the online channel.

Research limitations/implications: In this paper, we do not consider stochastic demand and asymmetric information, which may not well suit the reality.

Originality/value: This paper provides a different perspective to analyze the impact of supply chain power structures on the pricing decisions and online channel operation mode choice. The comparison of these two online channel operation modes in this paper is also a unique point. 
Keywords: supply chain management, power structures, online channel, game theory

\section{Introduction}

The past two decades has witnessed the popularity of the online electronic channels. Now more and more customers are accustomed to purchasing products online, which induces an increasing number of retailers or manufacturers to introduce the online channels into their traditional marketing channels. It is reported that on November 11th, 2014, the trade volume of Tmall.com, a platform for local Chinese and international businesses to sell brand name goods to consumers, is up to 35 billion Yuan. The convenience of online payment and prompt logistics distribution jointly contribute to the popularity online channels. Through online channels, firms can process customers' orders, control the distribution and pricing of goods, have a greater understanding of customers' preference (Mukhopadhyay, Zhu \& Yue, 2008). It is reported that about $42 \%$ of the top suppliers such as IBM, Nike, Pioneer Electronics, Estee Lauder, and Dell, are selling to customers through online channels (Chiang, Chhajed \& Hess, 2003; Tsay \& Agrawal, 2004).

Though the importance of introducing online channels has been widely recognized, which party should operate the online channels is not very clear. In practice, some manufacturers choose to open online channels of their own, while others mainly rely on their downstream retailers to operate the online channels. For example, Suning, one of the largest appliance retailers in China, sells the appliance both through the bricks and mortar and online channel. While Dell, one of the largest computer manufacturers in the world, operates both the retail channel and the online channel. A natural question emerges as for why different supply chains have different online channel operation modes? What factors impact the supply chain's online channel operation mode choice?

To answer these questions, we consider a two-echelon supply chain composed of one manufacturer and one retailer. Suppose that the supply chain is now considering introducing the online electronic channel. We consider three different supply chain power structures. In the manufacturer dominant market, the manufacturer acts as the Stackelberg leader and the retailer the follower. In the retailer dominant market, the retailer moves first as the Stackelberg leader and the manufacturer follows as the follower. If the manufacturer and the retailer have identical power, they move simultaneously. We compare the total profits of the supply chain under two different online channel operation modes: the manufacturer operating the online channel versus the retailer operating the online channel, under these three different supply chain power structures. We find that the online channel operation mode choice is jointly affected by customers' preference for the online channel, the self-price elasticity and the supply chain power structures. When the self-price elasticity is large, power structures have no direct impact on the online channel 
mode choice. However, when the self-price elasticity is small and customers' preference for the online channel is low, in the MS market, it is better for the retailer to operate the online channel, while in the RS and VN markets, it is better for the manufacturer to operate the online channel.

This paper falls into the dual-channel supply chain management. The channel structure composed of both the traditional offline retail channel and the online electronic channel is called the dual-channel. When the manufacturer opens the online channel, this phenomenon is called manufacturer encroachment (Arya, Mittendorf \& Sappington, 2007; Li, Gilbert \& Lai, 2013, 2015; Li, Xie \& Zhao, 2015). The manufacturer encroachment may cause severe channel conflict, because the manufacturer becomes the competitor of the downstream retailer. Some literature on dual-channel supply chain management focuses on the coordination mechanism to alleviate the potential channel conflict. Tsay and Agrawal (2004) study the channel conflict and coordination between the manufacturer and the retailer in a dual-channel supply chain. Mukhopadhyay et al. (2008) provides a way to alleviate the channel conflict when the retailer is able to add value to the product. Yan (2008) investigates the strategic role of profit sharing and finds that both parties will benefit from a dual-channel profit sharing strategy. Chiang et al. (2003) and Chen, Kaya and Özer (2008) study the pricing decisions between a manufacturer and a retailer in a dual-channel supply chain based on consumer choice behavior. Cai (2010) studies the influence of channel structures and pricing schemes on the dual-channel supply chain. Chen, Zhang and Sun (2012), Xu, Dan, Zhang and Liu (2014), study the coordination contract design in a manufacturer Stackelberg dual-channel supply chain.

Some literature introduces other decision variables into the dual-channel supply chain. Hua, Wang and Chen (2010) and Xu, Liu and Zhang (2012) study the pricing and lead time decisions in a dual-channel supply chain. Dan, Xu and Liu (2012) study the pricing and retail service decisions in a dual-channel supply chain. Huang, Yang and Zhang (2012) and Huang, Yang and Liu (2013) study the pricing and production decisions in a dual-channel supply chain when the supply chain experiences demand disruption and production cost disruption, respectively. Zhang, Xiong and Xiong (2015) study the coordination issue when a dual-channel supply chain experiences demand disruption or production cost disruption. Cao (2014) and Cao, Zhou and Lü (2015) study the channel coordination after demand disruption and production cost disruption. Cao, Ma, Wan and Lai (2013) study the contract design problem in a dual-channel supply chain with asymmetric cost information.

This paper is mostly related to the literature in dual-channel supply chain management when considering different supply chain power structures. Zhang, Liu and Wang (2012) study the effect of product substitutability and relative channel status on the optimal pricing decisions under different supply chain power structures. In their model, the online channel is operated by the manufacturer. Chen, Wang and Jiang (2015) investigate the impact of supply chain power structure on the 020 mixed dual-channel, where the retailer operates both the offline 
retail channel and the online electronic channel. Wang, Niu and Guo (2013) investigate a supply chain comprising an original equipment manufacturer and a contract manufacturer, where the contract manufacturer acts as both upstream partner and downstream competitor to the original equipment manufacturer. The two parties engage in one of three Cournot competition games: a simultaneous game, a sequential game with the original equipment manufacturer as the Stackelberg leader, and a sequential game with the contract manufacturer as the Stackelberg leader. Based on these three games, they investigate the two parties' Stackelberg leadership/followership decisions. Though there are already some literature addressing the pricing decisions under different supply chain power structures, there is little research on which supply chain party should operate the online channel. Because the online channel operation mode choice will affect the supply chain's optimal pricing decisions, which will eventually impact the optimal profits for different parties, it is necessary to incorporate the supply chain power structures into the supply chain's optimal online channel operation mode choice.

The rest of the paper is organized as follows. Section 2 presents the model setup. Section 3 analyzes the scenario of the manufacturer Stackelberg market. Section 4 analyzes the scenario of the retailer Stackelberg market. In section 5, the scenario of the vertical Nash market is analyzed. In the three different market structures, we derive the supply chain's optimal pricing decisions and the profits for different supply chain parties. At last, we compare the supplier chain's profits under two different online channel operation modes under different supply chain power structures. Section 6 summarizes the results.

\section{Model Setup}

Consider a supply chain composed of one manufacturer and one retailer. There are two selling channels, one is the traditional offline retail channel, and the other is the online electronic channel. In the offline retail channel, the manufacturer sells the product to the retailer at

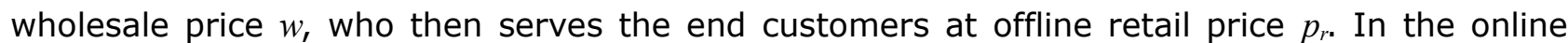
channel, we consider two kinds of online channel operation modes. In the first mode, the manufacturer operates the online channel and sells products directly to end customers at online retail price $p_{e}$, which is depicted in Figure 1 . This channel structure is also called the traditional dual-channel in operations management literature (Hua et al., 2010). The manufacturer simultaneously determines the wholesale price $w$ and the online retail price $p_{e}$ and the retailer determines the offline retail price $p_{r}$ 


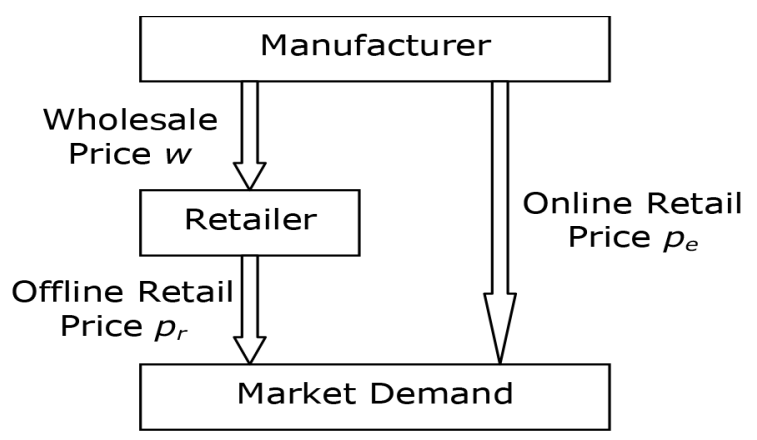

Figure 1. Manufacturer operates the online channel

In the second mode, the retailer operates the online channel and serves end customers through both the offline channel and the online channel, which is depicted in Figure 2 . This channel structure is also called the online-to-offline dual-channel in operations management literature (Chen et al., 2015). As more and more giant retailers choose to open online channels to sell products, this dual-channel structure is also very popular in practice. The manufacturer should determine the wholesale price $w$, and the retailer should simultaneously determine the offline retail price $p_{r}$ and the online retail price $p_{e}$. For analytical simplicity, following Arya et al. (2007) and Zhang et al. (2012), we normalize the unit production cost for the manufacturer and the selling cost to zeros.

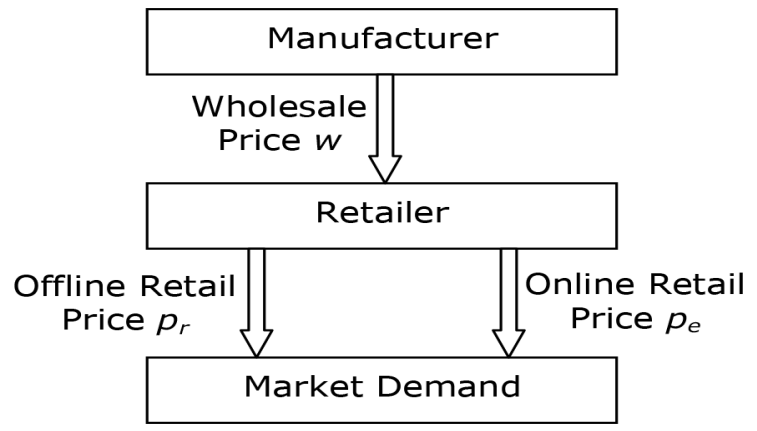

Figure 2. Retailer operates the online channel (Chen, Wang and Jiang, 2015)

For model simplicity, we assume that the demands in the two channels are linear in self-price and cross-price elasticity. This assumption is very common in operations management literature (Raju \& Abhik, 2000; Yue \& Liu, 2006; Kurata, Yao \& Liu, 2007; Huang \& Swaminathan, 2009; Hua et al., 2010; Huang et al., 2012; Huang et al., 2013; Chen et al., 2015). We further assume that the self-price elasticity and the cross-price elasticity are symmetric (Zhang et al., 2012; Chen et al., 2015).

The demand in the two channels, the offline channel and the online channel, are given by the following equations 


$$
\begin{gathered}
D_{r}\left(p_{r}, p_{e}\right)=(1-\rho) \theta-\alpha p_{r}+\beta p_{e} \\
D_{e}\left(p_{r}, p_{e}\right)=\rho \theta-\alpha p_{e}+\beta p_{r}
\end{gathered}
$$

where the subscript $r$ and $e$ denote the offline retail channel and the online electronic channel, respectively. The demand functions in Equations (1) and (2) indicate that the demands in the two channels both depend on the offline retail price $p_{r}$ and the online retail price $p_{e}$. The parameter $\theta$ represents the base market demand level. The parameter $\rho$ captures customers' preference for the online channel. The larger $\rho$ is, the more intense the channel conflict is (Hua et al., 2010; Huang et al., 2012). The parameter $\alpha$ is the coefficient of the self-price elasticity of its own channel, and the parameter $\beta$ is the coefficient of the cross-price elasticity of the opposite channel. Assume $\alpha>\beta$, so the price elasticity of the own channel is greater than the cross-price elasticity of the opposite channel (Hua et al., 2010; Huang et al., 2012).

We consider three different supply chain power structure models, i.e., the manufacturer Stackelberg model, the retailer Stackelberg model and the vertical Nash model. In the manufacturer Stackelberg model, the manufacturer moves first acting as the Stackelberg leader and then the retailer follows acting as the Stackelberg follower. In the retailer Stackelberg, the retailer moves first acting as the Stackelberg leader and then the manufacturer follows acting as the Stackelberg follower. In the vertical Nash model, both the manufacturer and the retailer move simultaneously. In the three different supply chain power structures, we consider two types of online channel operation modes, i.e., the manufacturer operating the online channel versus the retailer operating the online channel. Under these three different supply chain power structures, we concentrate on the analysis of the optimal pricing, as well as the comparison of the supply chain's performance, under the two online channel operation modes.

Our analysis is mostly related to Chen, Wang and Jiang (2015) and Huang, Yang and Zhang (2012), but with a different motivation. We would like to compare the performance of the two online channel operation modes and study which mode is superior. In order to make the paper self-contained, we use some results from Chen, Wang and Jiang (2015) and Huang, Yang and Zhang (2012) as the references. We list their results as lemmas throughout the paper.

\section{Manufacturer Stackelberg (MS) Model}

In this section, we consider the manufacturer Stackelberg market. In the MS market, the manufacturer acts as the Stackelberg leader and the retailer the Stackelberg follower. We consider two types of online channel operation modes, respectively. 


\subsection{The Manufacturer Operating the Online Channel}

The sequence of the events is as follows:

(1) The manufacturer first announces the wholesale price $w$ and the online retail price $p_{e}$;

(2) The retailer then announces the offline retail price $p_{r}$ given the manufacturer's pricing decisions;

(3) Finally, customer demand is resolved and the profits are realized according to the announced prices.

In the MS market with the manufacturer operating the online channel, the retailer's profit maximization problem is determined by

$$
\max \pi_{R}^{M S}\left(p_{r}\right)=\left(p_{r}-w\right)\left[(1-\rho) \theta-\alpha p_{r}+\beta p_{e}\right]
$$

and the manufacturer's profit function is determined by

$$
\max \pi_{M}^{M S}\left(w, p_{e}\right)=w\left[(1-\rho) \theta-\alpha p_{r}+\beta p_{e}\right]+p_{e}\left(\rho \theta-\alpha p_{e}+\beta p_{r}\right)
$$

where the subscript $R$ and $M$ denotes the retailer and manufacturer, respectively, throughout the paper. We solve this problem by backward induction. First, given the wholesale price $w$ and online retail price $p_{e}$ determined by the manufacturer, we obtain the retailer's optimal responsive pricing decision, and then we solve the manufacturer's optimal wholesale price $w$ and online retail price $p_{e}$.

The following lemma, which is presented in Huang, Yang and Zhang (2012), characterizes the optimal pricing decisions and profits in the MS market with the manufacturer operating the online channel.

Lemma 1 (Huang, Yang and Zhang, 2012). In the MS decentralized dual-channel supply chain with the manufacturer operating the online channel, the optimal pricing decisions are:

$$
\left\{\begin{array}{l}
w^{M S}=\frac{\alpha(1-\rho)+\beta \rho}{2\left(\alpha^{2}-\beta^{2}\right)} \theta \\
p_{r}^{M S}=\frac{2 \alpha \beta \rho+\left(3 \alpha^{2}-\beta^{2}\right)(1-\rho)}{4 \alpha\left(\alpha^{2}-\beta^{2}\right)} \theta \\
p_{e}^{M S}=\frac{\alpha \rho+\beta(1-\rho)}{2\left(\alpha^{2}-\beta^{2}\right)} \theta
\end{array}\right.
$$

And the supply chain's optimal profit is

$$
\pi_{S C}^{M S}=\frac{4 \alpha^{2} \rho^{2}+8 \alpha \beta \rho(1-\rho)+\left(3 \alpha^{2}+\beta^{2}\right)(1-\rho)^{2}}{16 \alpha\left(\alpha^{2}-\beta^{2}\right)} \theta^{2}
$$




\subsection{The Retailer Operating the Online Channel}

The sequence of the events is as follows:

(1) The manufacturer first announces the wholesale price $w$;

(2) The retailer then announces the offline retail price $p_{r}$ and the online retail price $p_{e}$, given the manufacturer's pricing decisions;

(3) Finally, customer demand is resolved and the profits are realized according to the announced prices.

In the MS market with the retailer operating the online channel, the retailer's profit function is determined by

$$
\Pi_{R}^{M S}\left(p_{r}, p_{e}\right)=\left(p_{r}-w\right)\left[(1-\rho) \theta-\alpha p_{r}+\beta p_{e}\right]+\left(p_{e}-w\right)\left(\rho \theta-\alpha p_{e}+\beta p_{r}\right)
$$

where the first term is the retailer's profit from the offline channel and the second term is the profit from the online channel.

And the manufacturer's profit function is determined by

$$
\Pi_{M}^{M S}(w)=w\left[\theta-(\alpha-\beta)\left(p_{r}+p_{e}\right)\right]
$$

The following lemma, presented in Chen, Wang and Jiang (2015), characterizes the optimal pricing decisions in the MS market with the retailer operating the online channel.

Lemma 2 (Chen, Wang and Jiang, 2015). In the MS decentralized dual-channel supply chain with the retailer operating the online channel, the optimal pricing decisions are:

$$
\left\{\begin{array}{l}
\bar{w}^{M S}=\frac{1}{4(\alpha-\beta)} \theta \\
\bar{p}_{r}^{M S}=\left[\frac{\alpha(1-\rho)+\beta \rho}{2\left(\alpha^{2}-\beta^{2}\right)}+\frac{1}{8(\alpha-\beta)}\right] \theta \\
\bar{p}_{e}^{M S}=\left[\frac{\alpha \rho+\beta(1-\rho)}{2\left(\alpha^{2}-\beta^{2}\right)}+\frac{1}{8(\alpha-\beta)}\right] \theta
\end{array}\right.
$$

And the supply chain's optimal profit is

$$
\Pi_{S C}^{M S}=\left[\frac{\alpha \rho^{2}+2 \beta \rho(1-\rho)+\alpha(1-\rho)^{2}}{4\left(\alpha^{2}-\beta^{2}\right)}-\frac{1}{32(\alpha-\beta)}\right] \theta^{2}
$$




\subsection{Comparison Analysis}

The above two subsections analyze the optimal pricing decisions and the supply chain performances for the two online channel operation modes, respectively. In subsequent, we would like to analyze, from the perspective of the supply chain, which online channel operation mode is more efficient in the MS market. The following proposition characterizes the result.

Proposition 1. If $0<\rho<1-\sqrt{\alpha /[2(\alpha-\beta)]}$, then $\Pi_{S C}^{M S}>\pi_{S C}^{M S}$, otherwise, $\Pi_{S C}^{M S} \leq \pi_{S C}^{M S}$.

Proof. From the results in Lemma 1 and Lemma 2, we have

$$
\begin{aligned}
\Pi_{S C}^{M S}-\pi_{S C}^{M S} & =\left[\frac{\alpha \rho^{2}+2 \beta \rho(1-\rho)+\alpha(1-\rho)^{2}}{4\left(\alpha^{2}-\beta^{2}\right)}-\frac{1}{32(\alpha-\beta)}\right] \theta^{2}-\left[\frac{2 \alpha^{2} \rho^{2}+4 \alpha \beta \rho(1-\rho)+\left(\alpha^{2}+\beta^{2}\right)(1-\rho)^{2}}{8 \alpha\left(\alpha^{2}-\beta^{2}\right)}+\frac{(1-\rho)^{2}}{16 \alpha}\right] \theta^{2} \\
& =\frac{2(\alpha-\beta)(1-\rho)^{2}-\alpha}{32 \alpha(\alpha-\beta)} \theta^{2}
\end{aligned}
$$

If the customers' preference for the online channel satisfy $0<\rho<1-\sqrt{\alpha /[2(\alpha-\beta)]}$, then $2(\alpha-\beta)(1-\rho)^{2}>\alpha$, otherwise, $2(\alpha-\beta)(1-\rho)^{2} \leq \alpha$. Thus, the results can be easily obtained.

The following corollary characterizes the impact of customers' preference on the online channel on the supply chain's optimal pricing decisions.

Corollary 1. (1) $\bar{p}_{e}^{M S}>p_{e}^{M S}$; (2) $\bar{w}^{M S} \geq w^{M S}$ if and only if $\rho \geq 1 / 2$; (3) $\bar{p}_{r}^{M S}>p_{r}^{M S}$ if and only if $\rho>\{(\alpha-2 \beta) /[2(\alpha-\beta)]\}^{+}$.

Proof. Comparing the results in Lemma 1 and Lemma 2, we have: (1) $\bar{p}_{e}^{M S}-p_{e}^{M S}=\frac{\theta}{8(\alpha-\beta)}>0$; (2) $\bar{w}^{M S}-w^{M S}=\frac{2 \rho-1}{4(\alpha+\beta)} \theta$, which is nonnegative if an only if $\rho \geq 1 / 2 ;(3) \bar{p}_{r}^{M S}-p_{r}^{M S}=\frac{2(\alpha-\beta) \rho-(\alpha-2 \beta)}{8 \alpha(\alpha-\beta)} \theta$, then the results can be easily obtained.

Proposition 1 indicates that, in the MS market, if customers' preference for the online channel is low, then from the perspective of the supply chain, it is better for the manufacturer to operate the online channel. Otherwise, it is better for the retailer to operate the online channel. The intuition is as follows. When customers' preference for the online channel is low, the retailer knows more about customers' channel preference and is in an ideal position to introduce the online channel to the customers. For example, when a customer goes into Suning Appliance to buy a refrigerator, obviously, he may not know the possibility of buying appliances online or just prefer to buy on spot. In order to encourage the customer to purchase online at suning.com, Suning offers customers coupons which can be used for other future orders made online at suning.com. In other words, retailers can make full use of the advantage of contacting customers to introduce the online channel. When customers' preference for the online channel is high, the manufacturer should operates the online channel, this is because from Corollary 1 we know that, when $\rho$ is large, $\bar{p}_{e}^{M S}>p_{e}^{M S}$, which means that the 
optimal online retail price under manufacturer operating the online channel is smaller than that under retailer operating the online channel. It is better for the manufacturer to operate the online channel.

\section{Retailer Stackelberg (RS) Model}

In this section, we analyze the retailer Stackelberg market. In the RS market, the retailer acts as the Stackelberg leader and the manufacturer acts as the Stackelberg follower. Similar to Section 3, we analyze the optimal pricing decisions and supply chain performance under two decision modes.

\subsection{The Manufacturer Operating the Online Channel}

The sequence of the evens is as follows:

(1) The retailer first announces the offline retailer price $p_{r}$;

(2) The manufacturer then announces the online retail price $p_{e}$ and the wholesale price $w$, given the retailer's pricing decision;

(3) Finally, customer demand is resolved and the profits are realized according to the announced prices.

Following Zhang et al. (2012) and Chen et al., 2015, we assume the marginal profit for the offline channel is $m_{r}=p_{r}-w$. In the RS market with the manufacturer operating the online channel, the manufacturer's profit, denoted by $\pi_{M}^{R S}\left(w, p_{e}\right)$, is

$$
\pi_{M}^{R S}\left(w, p_{e}\right)=w\left[(1-\rho) \theta-\alpha\left(m_{r}+w\right)+\beta p_{e}\right]+p_{e}\left[\rho \theta-\alpha p_{e}+\beta\left(m_{r}+w\right)\right]
$$

In Equation (12), the first term is the profit from the offline channel, and the second term is the profit from the online channel. The retailer's profit, denoted by $\pi_{R}^{R S}\left(p_{r}\right)$, is

$$
\pi_{R}^{R S}\left(p_{r}\right)=\left(p_{r}-w\right)\left[(1-\rho) \theta-\alpha p_{r}+\beta p_{e}\right]
$$

The following proposition characterizes the optimal pricing decisions and profits in the RS market with the manufacturer operating the online channel.

Proposition 2. In the RS decentralized dual-channel supply chain with the manufacturer operating the online channel, the optimal pricing decisions are: 


$$
\left\{\begin{array}{l}
w^{R S}=\frac{2 \alpha \beta \rho+\left(\alpha^{2}+\beta^{2}\right)(1-\rho)}{4 \alpha\left(\alpha^{2}-\beta^{2}\right)} \theta \\
p_{r}^{R S}=\frac{2 \alpha \beta \rho+\left(3 \alpha^{2}-\beta^{2}\right)(1-\rho)}{4 \alpha\left(\alpha^{2}-\beta^{2}\right)} \theta \\
p_{e}^{R S}=\frac{\alpha \rho+\beta(1-\rho)}{2\left(\alpha^{2}-\beta^{2}\right)} \theta
\end{array}\right.
$$

And the supply chain's optimal profit is

$$
\pi_{S C}^{R S}=\frac{4 \alpha^{2} \rho^{2}+8 \alpha \beta \rho(1-\rho)+\left(3 \alpha^{2}+\beta^{2}\right)(1-\rho)^{2}}{16 \alpha\left(\alpha^{2}-\beta^{2}\right)} \theta^{2}
$$

Proof. Taking the first-order and second-order derivatives of $\pi_{M}^{R S}\left(w, p_{e}\right)$ with respect to $w$ and $p_{e}$ respectively, we obtain

$$
\begin{gathered}
\partial \pi_{M}^{R S} / \partial w=(1-\rho) \theta-\alpha m_{r}-2 \alpha w+2 \beta p_{e} \\
\partial \pi_{M}^{R S} / \partial p_{e}=\rho \theta+\beta m_{r}+2 \beta w-2 \alpha p_{e} \\
\partial^{2} \pi_{M}^{R S} / \partial w^{2}=\partial^{2} \pi_{M}^{R S} / \partial p_{e}^{2}=-2 \alpha<0 \\
\partial^{2} \pi_{M}^{R S} / \partial w \partial p_{e}=\partial^{2} \pi_{M}^{R S} / \partial p_{e} \partial w=2 \beta>0
\end{gathered}
$$

It is easy to verify that $\pi_{M}^{R S}\left(w, p_{e}\right)$ is jointly concave in $w$ and $p_{e}$. Let $\partial \pi_{M}^{R S} / \partial w=0$ and $\partial \pi_{M}^{R S} / \partial p_{e}=0$, we get $p_{e}^{R S}=\frac{\alpha \rho+\beta(1-\rho)}{2\left(\alpha^{2}-\beta^{2}\right)} \theta$ and $w=\frac{\alpha(1-\rho)+\beta \rho}{\alpha^{2}-\beta^{2}} a-p_{r}$. Then the retailer's decision problem can be rewritten as

$$
\max \pi_{R}^{R S}\left(p_{r}\right)=\left[2 p_{r}-\frac{\alpha(1-\rho)+\beta \rho}{\alpha^{2}-\beta^{2}} \theta\right]\left[(1-\rho) \theta-\alpha p_{r}+\beta \frac{\alpha \rho+\beta(1-\rho)}{2\left(\alpha^{2}-\beta^{2}\right)} \theta\right]
$$

Since $\partial^{2} \pi_{R}^{R S} / \partial p_{r}^{2}=-4 \alpha<0$, which indicates that $\pi_{R}^{R S}\left(p_{r}\right)$ is concave in $p_{r}$, then from the firstorder condition $\partial \pi_{R}^{R S} / \partial p_{r}=-4 \alpha p_{r}+\frac{2 \alpha \beta \rho+\left(3 \alpha^{2}-\beta^{2}\right)(1-\rho)}{\alpha^{2}-\beta^{2}} \theta=0$, we get $p_{r}^{R S}$. Substituting it into $w=\frac{\alpha(1-\rho)+\beta \rho}{\alpha^{2}-\beta^{2}} a-p_{r}$, we obtain $w^{R S}$.

\subsection{The Retailer Operating the Online Channel}

The sequence of the events is as follows:

(1) The retailer first announces offline retail price $p_{r}$ and the online retail price $p_{e}$;

(2) The manufacturer then announces the wholesale price $w$, given the retailer's pricing decisions; 
(3) Finally, customer demand is resolved and the profits are realized according to the announced prices.

The following proposition, presented in Chen, Wang and Jiang (2015), characterizes the optimal pricing decisions in the RS market with the retailer operating the online channel.

Lemma 3 (Chen, Wang and Jiang, 2015). In the RS decentralized dual-channel supply chain with the retailer operating the online channel, the optimal pricing decisions are:

$$
\left\{\begin{array}{l}
\bar{w}^{R S}=\frac{1}{8(\alpha-\beta)} \theta \\
\bar{p}_{r}^{R S}=\frac{(\alpha+5 \beta) \rho+(5 \alpha+\beta)(1-\rho)}{8\left(\alpha^{2}-\beta^{2}\right)} \theta \\
\bar{p}_{e}^{R S}=\frac{(5 \alpha+\beta) \rho+(\alpha+5 \beta)(1-\rho)}{8\left(\alpha^{2}-\beta^{2}\right)} \theta
\end{array}\right.
$$

And the supply chain's optimal profit is

$$
\Pi_{S C}^{R S}=\frac{(7 \alpha-\beta) \rho^{2}-2(\alpha-7 \beta) \rho(1-\rho)+(7 \alpha-\beta)(1-\rho)^{2}}{32\left(\alpha^{2}-\beta^{2}\right)} \theta^{2}
$$

\subsection{Comparison Analysis}

We next compare the supply chain's profit under these two online channel operation modes in the RS market. The following proposition characterizes the result.

Proposition 3. (1) If $\beta<\alpha \leq 2 \beta$, then $\Pi_{S C}^{R S} \leq \pi_{S C}^{R S}$; (2) If $\alpha \geq 2 \beta$, then $\Pi_{S C}^{R S}>\pi_{S C}^{R S}$ if and only if $0<\rho<1-\sqrt{\alpha /[2(\alpha-\beta)]}$.

Proof. From the results in Lemma 3 and Proposition 2, we have

$$
\begin{aligned}
\Pi_{S C}^{R S}-\pi_{S C}^{R S} & =\frac{(7 \alpha-\beta) \rho^{2}-2(\alpha-7 \beta) \rho(1-\rho)+(7 \alpha-\beta)(1-\rho)^{2}}{32\left(\alpha^{2}-\beta^{2}\right)} \theta^{2}-\frac{4 \alpha^{2} \rho^{2}+8 \alpha \beta \rho(1-\rho)+\left(3 \alpha^{2}+\beta^{2}\right)(1-\rho)^{2}}{16 \alpha\left(\alpha^{2}-\beta^{2}\right)} \theta^{2} \\
& =\frac{\theta^{2}}{32 \alpha}\left(2 \rho^{2}-4 \rho+1-\frac{\beta}{\alpha-\beta}\right)
\end{aligned}
$$

Let $H(\rho)=2 \rho^{2}-4 \rho+1-\frac{\beta}{\alpha-\beta}$, which is quadratic and convex in $\rho \in(0,1)$ and minimized when $\rho \rightarrow 1$. Thus, we can obtain the results by analyzing the property of the function of $H(\rho)$ in three different cases.

Proposition 3 indicates that when the self-price elasticity is small, it is better for the manufacturer to operate the online channel. However, when the self-price elasticity is large, then if customers' preference for the online channel is low, it is better for the retailer to operate the online channel, otherwise, it is better for the manufacturer to operate the 
online channel. The intuition is as follows. Since the self-price elasticity is not too large compared with the cross-price elasticity, which means that the channel substitution is high, in this case, the retailer will take mild actions to cope with the introduction of the online channel. If the retailer operates the online channel, the impact of channel encroachment will has more direct adverse impact on the retailer's profit. Thus, the manufacturer operating the online channel will be beneficial to both parties. However, when the sell-price elasticity is large, this means that the channel substitution is low. In this case, if customers' preference for the online channel is low, then the retailer can make full use of her advantage of contacting customers to introduce the online channel, which will make the online channel more profitable. If the manufacturer operates the online channel, then the recognition degree of the online channel will be low and the online channel will be not efficient enough. On the contrary, if customers' preference for the online channel is high, then the manufacturer should operate the online channel because he has the cost advantage.

\section{Vertical Nash (VN) Model}

In subsequent, we would like to analyze the vertical Nash market. In the VN market, the manufacturer and the retailer are in identical power position. They move simultaneously to maximize their individual profits without knowing the other party's pricing decisions. Similarly, we analyze the optimal pricing decisions and supply chain performance under two decision modes.

\subsection{The Manufacturer Operating the Online Channel}

The sequence of the evens is as follows:

(1) The retailer announces the offline retailer price $p_{r}$, meanwhile, the manufacturer announces the wholesale price $w$ and the online retail price $p_{e} ;$

(2) Then customer demand is resolved and the profits are realized according to the announced prices.

Assume that the marginal profit for the offline channel is $m_{r}=p_{r}-w$. In the VN market with the manufacturer operating the online channel, the manufacturer's profit, denoted by $\pi_{M}^{i N}\left(w, p_{e}\right)$, is

$$
\pi_{M}^{V N}\left(w, p_{e}\right)=w\left[(1-\rho) \theta-\alpha\left(m_{r}+w\right)+\beta p_{e}\right]+p_{e}\left[\rho \theta-\alpha p_{e}+\beta\left(m_{r}+w\right)\right]
$$

where the first term is the profit from the offline channel, and the second term is the profit from the online channel. 
And the retailer's profit, denoted by $\Pi_{R}^{V N}\left(p_{r}\right)$, is

$$
\pi_{R}^{V N}\left(p_{r}\right)=\left(p_{r}-w\right)\left[(1-\rho) \theta-\alpha p_{r}+\beta p_{e}\right]
$$

The following proposition characterizes the optimal pricing decisions and profits in the VN market with the manufacturer operating the online channel.

Proposition 4. In the VN decentralized dual-channel supply chain with the manufacturer operating the online channel, the optimal pricing decisions are:

$$
\left\{\begin{array}{l}
w^{V N}=\frac{3 \alpha \beta \rho+\left(2 \alpha^{2}+\beta^{2}\right)(1-\rho)}{6 \alpha\left(\alpha^{2}-\beta^{2}\right)} \theta \\
p_{r}^{V N}=\frac{3 \alpha \beta \rho+\left(4 \alpha^{2}-\beta^{2}\right)(1-\rho)}{6 \alpha\left(\alpha^{2}-\beta^{2}\right)} \theta \\
p_{e}^{V N}=\frac{\alpha \rho+\beta(1-\rho)}{2\left(\alpha^{2}-\beta^{2}\right)} \theta
\end{array}\right.
$$

And the supply chain's optimal profit is

$$
\pi_{S C}^{V N}=\frac{9 \alpha^{2} \rho^{2}+18 \alpha \beta \rho(1-\rho)+\left(8 \alpha^{2}+\beta^{2}\right)(1-\rho)^{2}}{36 \alpha\left(\alpha^{2}-\beta^{2}\right)} \theta^{2}
$$

Proof. From Equation (24), we have

$$
\begin{gathered}
\partial \pi_{M}^{V N} / \partial w=(1-\rho) \theta-\alpha m_{r}-2 \alpha w+2 \beta p_{e} \\
\partial \pi_{M}^{V N} / \partial p_{e}=\rho \theta+\beta m_{r}-2 \alpha p_{e}+2 \beta w \\
\partial^{2} \pi_{M}^{V N} / \partial w^{2}=\partial^{2} \pi_{M}^{V N} / \partial p_{e}^{2}=-2 \alpha<0 \\
\partial^{2} \pi_{M}^{V N} / \partial w \partial p_{e}=\partial^{2} \pi_{M}^{V N} / \partial p_{e} \partial w=2 \beta
\end{gathered}
$$

It can be verified that $\pi_{M}^{V N}\left(w, p_{e}\right)$ is jointly concave in $w$ and $p_{e}$. Let $\partial \pi_{M}^{V N} / \partial w=0$ and $\partial \pi_{M}^{V N} / \partial p_{e}=0$, we obtain $p_{e}^{V N}=\frac{\alpha \rho+\beta(1-\rho)}{2\left(\alpha^{2}-\beta^{2}\right)} \theta$ and $w=\frac{\alpha(1-\rho)+\beta \rho}{2\left(\alpha^{2}-\beta^{2}\right)} \theta-\frac{p_{r}-w}{2}$, which jointly reduces to $w=\frac{\alpha(1-\rho)+\beta \rho}{\alpha^{2}-\beta^{2}} \theta-p_{r}$. Let $\partial \pi_{R}^{V N} / \partial p_{r}=0$, we get $p_{r}=\frac{1}{2 \alpha}\left[(1-\rho) \theta+\beta \frac{\alpha \rho+\beta(1-\rho)}{2\left(\alpha^{2}-\beta^{2}\right)} \theta+\alpha w\right]$, thus, we can obtain $w^{V N}$ and $p_{r}^{V N}$.

\subsection{The Retailer Operating the Online Channel}

The sequence of the events is as follows:

(1) The retailer announces the offline retailer price $p_{r}$ and the online retail price $p_{e}$ meanwhile, the manufacturer announces the wholesale price $w$; 
(2) Then customer demand is resolved and the profits are realized according to the announced prices.

The following lemma, presented in Chen, Wang and Jiang (2015), characterizes the optimal pricing decisions in the VN market with the retailer operating the online channel.

Lemma 5 (Chen, Wang and Jiang, 2015). In the VN decentralized dual-channel supply chain with the retailer operating the online channel, the optimal pricing decisions are:

$$
\left\{\begin{array}{l}
\bar{w}^{I N}=\frac{1}{6(\alpha-\beta)} \theta \\
\bar{p}_{r}^{V N}=\frac{(\alpha+7 \beta) \rho+(7 \alpha+\beta)(1-\rho)}{12\left(\alpha^{2}-\beta^{2}\right)} \theta \\
\bar{p}_{e}^{V N}=\frac{(7 \alpha+\beta) \rho+(\alpha+7 \beta)(1-\rho)}{12\left(\alpha^{2}-\beta^{2}\right)} \theta
\end{array}\right.
$$

And the supply chain's optimal profit is

$$
\Pi_{S C}^{V N}=\frac{(17 \alpha-\beta) \rho^{2}+2(17 \beta-\alpha) \rho(1-\rho)+(17 \alpha-\beta)(1-\rho)^{2}}{72\left(\alpha^{2}-\beta^{2}\right)} \theta^{2}
$$

\subsection{Comparison Analysis}

We next compare the supply chain's profits under these two online channel operation modes in the VN market. The following proposition characterizes the result.

Proposition 5. (1) If $\beta<\alpha \leq 2 \beta$, then $\Pi_{S C}^{V N} \leq \pi_{S C}^{V N}$; (2) If $\alpha>2 \beta$, then $\Pi_{S C}^{V N}>\pi_{S C}^{V N}$ if and only if $0<\rho<1-\sqrt{\alpha /[2(\alpha-\beta)]}$.

Proof. From the results in Proposition 4 and Lemma 5, we have

$$
\begin{aligned}
\Pi_{S C}^{N V}-\pi_{S C}^{N V} & =\frac{(17 \alpha-\beta) \rho^{2}+2(17 \beta-\alpha) \rho(1-\rho)+(17 \alpha-\beta)(1-\rho)^{2}}{72\left(\alpha^{2}-\beta^{2}\right)} \theta^{2}-\frac{9 \alpha^{2} \rho^{2}+18 \alpha \beta \rho(1-\rho)+\left(8 \alpha^{2}+\beta^{2}\right)(1-\rho)^{2}}{36 \alpha\left(\alpha^{2}-\beta^{2}\right)} \theta^{2} \\
& =\frac{\theta^{2}}{72 \alpha}\left(2 \rho^{2}-4 \rho+1-\frac{\beta}{\alpha-\beta}\right)
\end{aligned}
$$

Let $H(\rho)=2 \rho^{2}-4 \rho+1-\frac{\beta}{\alpha-\beta}$, which is quadratic in $\rho \in(0,1)$ and achieving the minimum when $\rho \rightarrow 1$. We can easily obtain the results by analyzing the property of the function $H(\rho)$.

Interestingly, the results in Proposition 5 are somewhat similar to those in the Proposition 6, though the optimal pricing decisions and the optimal profits for the supply chain in the RS market and the VN market are completely different. However, the profit differentials for the supply chain under the two supply chain power structures are similar. Specifically, $9\left(\Pi_{S C}^{N V}-\pi_{S C}^{N V}\right)=4\left(\Pi_{S C}^{R S}-\pi_{S C}^{R S}\right)$. 
We next summarize the main results in Proposition 1, Proposition 3 and Proposition 5 in the following Table 1.

\begin{tabular}{|c|c|c|c|c|}
\hline$\alpha$ & $\rho$ & MS & RS & VN \\
\hline$\beta<\alpha \leq 2 \beta$ & $0<\rho<1-\sqrt{\alpha /[2(\alpha-\beta)]}$ & Retailer & Manufacturer & Manufacturer \\
\hline$\beta<\alpha \leq 2 \beta$ & $1-\sqrt{\alpha /[2(\alpha-\beta)]} \leq \rho<1$ & Manufacturer & Manufacturer & Manufacturer \\
\hline$\alpha>2 \beta$ & $0<\rho<1-\sqrt{\alpha /[2(\alpha-\beta)]}$ & Retailer & Retailer & Retailer \\
\hline$\alpha>2 \beta$ & $1-\sqrt{\alpha /[2(\alpha-\beta)]} \leq \rho<1$ & Manufacturer & Manufacturer & Manufacturer \\
\hline
\end{tabular}

Table 1. Manufacturer vs. retailer operating the online channel in different power structures

From Table 1, we find that the online channel operation mode is affected by customers' preference for the online channel, the self-price elasticity and the supply chain power structures. When the self-price elasticity is large, the supply chain power structure has no impact on the online channel model. Only customers' preference for the online channel impacts the supply chain's optimal decision. When customers' preference for the online channel is low, it is better for the retailer to operate the online channel, otherwise, it is better for the manufacturer to operate the online channel. However, when the self-price elasticity is small, then if customers' preference for the online channel is high, then it's better for the manufacturer to operate the online channel, no matter what the supply chain power structure is. However, when customers' preference for the online channel is low, then in the MS market, it is better for the retailer to operate the online channel, while in the RS market and the VN market, it is better for the manufacturer to operate the online channel.

The results in Table 1 imply that the two channel structures, RS and VN, have no direct impact on the online channel operation mode choice. However, it does convey some important messages. When the retailers are more powerful or equal powerful compared with the manufacturers, most of the time the online channel should be operated by the manufacturers. This may be an efficient way to alleviate the double marginalization effect caused by decentralized decisions. But this is not always the case, especially when the cross-price effect is low and customers' preference for the online channel is low. At this time, the retailer should operate the online channel by herself. Therefore, when a manager plans to introduce the online channel into the extant channel configuration, he should simultaneously consider three key factors, customers' preference for the online channel, the adverse impact of the online channel on the demand of the traditional retail channel, as well as the supply chain power between the retailer and the manufacturer. The retailer should be very cautious to open an online channel and operate it by herself. Because in most cases, the operation efficiency of the retailer operating the online channel mode is lower than that of the manufacturer operating the online 
channel mode. This is parallel with real business situations. Though some retailers have taken on the online-to-offline channel strategy, however, in practice, most of the online channels are operated by the manufacturers. The results in Table 1 may give an explanation why most of the online channels are operated by the manufacturers.

\section{Concluding Remarks}

The introduction of online channel can be beneficial to the supply chain, because it can reach more customers, which cannot be achieved by the traditional single channel. Hence, more and more supply chain systems have been introducing the online channels into their traditional supply chain channel configurations. However, thought there is much literature on how to price a dual-channel supply, how to coordinate the dual supply chain, as well as how to improve the dual-channel supply chain with other performance-improving considerations, the question on which party should operate the online channel is not well addressed. In this paper, we address the online channel operation mode decision, i.e., when considering opening an online electronic channel, which party should operate the online channel. We consider the problem from the perspective of supply chain power structures. We consider three different supply chain power structures, which are the manufacturer Stackelberg, the retailer Stackelberg and the vertical Nash. By using the noncooperative game theory, we obtain the optimal pricing decisions and optimal profits for the manufacturer, the retailer and the supply chain. We then compare the supply chain's total profits under different scenarios.

We find that the online channel operation mode is significantly affected by customers' preference for the online channel and the self-price elasticity in different supply chain power structures. When the self-price elasticity is very large, if customer's preference for the online channel is low, then it is better for the retailer to operate the online channel, otherwise, it is better for the manufacturer to operate the online channel. However, when the self-price elasticity is small, then if customers' preference for the online channel is high, then it's better for the manufacturer to operate the online channel. However, when customers' preference for the online channel is low, then in the MS market, it is better for the retailer to operate the online channel, while in the RS market and in the VN market, it is better for the manufacturer to operate the online channel.

The contributions of the paper are two-fold. On the one hand, the results in this paper may help business managers to make proper online channel operation mode decisions. When making decisions as for introducing an online channel, the decision-maker should take into considerations the supply chain power structures, customers' preference for the online channel and the cross-price elasticity. Ignorance of one of these factors may lead to suboptimal decisions. On the other hand, the optimal pricing decisions are also presented in the paper, and the supply parties' optimal profits, as well as the total supply chain's profit, are obtained. In 
practice, we find that there are some supply chains with the retailers operating the online channel, while others with the manufacturers operating the online channel, however, the underlying reason is not yet very clear. The results of this paper may give some analytical explanations to some extent. Admittedly, the results obtained in this paper are based on some stylized game theoretical models which only capture some important parameters in real situations. In practice, as for which party should operate the online channel, there are some other factors that must also be taken into consideration, such as the experience of channel management, the investment of information infrastructure, and some specific properties of the products, and so on. It seems more benefiting to incorporate these factors into the stylized game theoretical modes, and sometimes this may leads to intractable models. Consequently, we choose to focus on the impact of supply chain power structures on the online channel operation mode choice.

Some extensions are possible to conduct based on this paper. First, the scenario of stochastic demand can be incorporated into the current research framework. Second, the asymmetric information about the market demand can also be considered, and some interesting results may be obtained. Third, the risk attitude of the supply chain parties can be further considered. These extensions are interesting work in the future.

\section{Acknowledgements}

This research is supported in part by the National Natural Science Foundation of China under Grant 71301055, The Youth Fund of Humanities and Social Science of Ministry of Education of China (13YJC630054), and Natural Science Foundation of Guangdong Province, China (S2013040016248).

\section{References}

Arya, A., Mittendorf, B., \& Sappington, D.E.M. (2007). The bright side of supplier encroachment. Marketing Science, 26(5), 651-659. http://dx.doi.org/10.1287/mksc.1070.0280

Cai, G. (2010). Channel selection and coordination in dual-channel supply chains. Journal of Retailing, 86(1), 22-36. http://dx.doi.org/10.1016/j.jretai.2009.11.002

Cao, E. (2014). Coordination of dual-channel supply chains under demand disruptions management decisions. International Journal of Production Research, 52(23), 7114-7131. http://dx.doi.org/10.1080/00207543.2014.938835 
Cao, E., Zhou, X., \& Lü, K. (2015). Coordinating a supply chain under demand and cost disruptions. International Journal of Production Research, 53(12), 3735-3752. http://dx.doi.org/10.1080/00207543.2014.988885

Cao, E., Ma, Y., Wan, C., \& Lai, M. (2013). Contracting with asymmetric cost information in a dual-channel supply chain. Operations Research Letters, 41(4), 410-414. http://dx.doi.org/10.1016/j.orl.2013.04.013

Chen, J., Zhang, H., \& Sun, Y. (2012). Implementing coordination contracts in a manufacturer Stackelberg dual-channel supply chain. Omega, 40(5), 571-583. http://dx.doi.org/10.1016/j.omega.2011.11.005

Chen, K.Y., Kaya, M., \& Özer, Ö. (2008). Dual sales channel management with service competition. Manufacturing \& Service Operations Management, 10(4), 654-675. http://dx.doi.org/10.1287/msom.1070.0177

Chen, X., Wang, X., \& Jiang, X. (2015). The impact of power structure on the retail service supply chain with an 020 mixed channel. Journal of the Operational Research Society. http://dx.doi.org/10.1057/jors.2015.6

Chiang, W.K., Chhajed, D., \& Hess, J.D. (2003). Direct marketing, indirect profits: a strategic analysis of dual channel supply-chain design. Management Science, 49(1), 1-20. http://dx.doi.org/10.1287/mnsc.49.1.1.12749

Dan, B., Xu, G., \& Liu, C. (2012). Pricing policies in a dual-channel supply chain with retail services. International Journal of Production Economics, 139(1), 312-320. http://dx.doi.org/10.1016/j.ijpe.2012.05.014

Hua, G., Wang, S., \& Cheng, T.C.E. (2010). Price and lead time decisions in dual-channel supply chains. European Journal of Operational Research, 205(1), 113-126. http://dx.doi.org/10.1016/j.ejor.2009.12.012

Huang, S., Yang, C., \& Liu, H. (2013). Pricing and production decisions in a dual-channel supply chain when production costs are disrupted. Economic Modelling, 30, 521-538. http://dx.doi.org/10.1016/j.econmod.2012.10.009

Huang, S., Yang, C., \& Zhang, X. (2012). Pricing and production decisions in dual-channel supply chains with demand disruptions. Computers \& Industrial Engineering, 62(1), 70-83. http://dx.doi.org/10.1016/j.cie.2011.08.017

Huang, W., \& Swaminathan, J.M. (2009). Introduction of a second channel: Implications for pricing and profits. European Journal of Operational Research, 194(1), 258-279. http://dx.doi.org/10.1016/j.ejor.2007.11.041

Kurata, H., Yao, D.Q., \& Liu, J.J. (2007). Pricing polices under direct vs. indirect channel competition and national vs. store brand competition. European Journal of Operational Research, 180(1), 262-281. http://dx.doi.org/10.1016/j.ejor.2006.04.002 
Li, T., Xie, J., \& Zhao, X. (2015). Supplier encroachment in competitive supply chains. International Journal of Production Economics, 165, 120-131. http://dx.doi.org/10.1016/j.ijpe.2015.03.023

Li, Z., Gilbert, S.M., \& Lai, G. (2013) Supplier encroachment under asymmetric information. Management Science, 60(2), 449-462. http://dx.doi.org/10.1287/mnsc.2013.1780

Li, Z., Gilbert, S.M., \& Lai, G. (2015). Supplier encroachment as an enhancement or a hindrance to nonlinear pricing. Production and Operations Management, 24(1), 89-109. http://dx.doi.org/10.1111/poms.12210

Mukhopadhyay, S.K., Zhu, X., \& Yue, X. (2008). Optimal contract design for mixed channels under information asymmetry. Production and Operations Management, 17(6), 641-650. http://dx.doi.org/10.3401/poms.1080.0069

Raju, J., \& Abhik, R. (2000). Market information and firm performance. Management Science, 46(8), 1075-1084. http://dx.doi.org/10.1287/mnsc.46.8.1075.12024

Tsay, A., \& Agrawal, N. (2004). Channel conflict and coordination in the E-commerce age. Production and Operations Management, 13(1), 93-110. http://dx.doi.org/10.1111/j.19375956.2004.tb00147.x

Wang, Y., Niu, B., \& Guo, P. (2013). On the advantage of quantity leadership when outsourcing production to a competitive contract manufacturer. Production and Operations Management, 22(1), 104-119. http://dx.doi.org/10.1111/j.1937-5956.2012.01336.x

Xu, G., Dan, B., \& Zhang, X., \& Liu, C. (2014). Coordinating a dual-channel supply chain with risk-averse under a two-way revenue sharing contract. International Journal of Production Economics, 147, 171-179. http://dx.doi.org/10.1016/j.ijpe.2013.09.012

Xu, H., Liu, Z.Z., \& Zhang, S.H. (2012). A strategic analysis of dual-channel supply chain design with price and delivery lead time considerations. International Journal of Production Economics, 139(2), 654-663. http://dx.doi.org/10.1016/j.ijpe.2012.06.014

Yan, R. (2008). Profit sharing and firm performance in the manufacturer-retailer dual-channel supply chain. Electronic Commerce Research, 8(3): 155-172. http://dx.doi.org/10.1007/s10660-0089020-2

Yue, X., \& Liu, J.J. (2006). Demand forecast sharing in a dual-channel supply chain. European Journal of Operational Research, 174(1), 646-667. http://dx.doi.org/10.1016/j.ejor.2004.12.020

Zhang, P., Xiong, Y., \& Xiong, Z. (2015). Coordination of a dual-channel supply chain after demand or production cost disruptions. International Journal of Production Research, 53(10): 3141-3160. http://dx.doi.org/10.1080/00207543.2014.975853 
Zhang, R., Liu, B., \& Wang, W. (2012). Pricing decisions in a dual channel system with different power structures. Economic Modelling, 29(2), 523-533.

http://dx.doi.org/10.1016/j.econmod.2011.08.024

Journal of Industrial Engineering and Management, 2015 (www.jiem.org)

Article's contents are provided on an Attribution-Non Commercial 3.0 Creative commons license. Readers are allowed to copy, distribute and communicate article's contents, provided the author's and Journal of Industrial Engineering and Management's names are included.

It must not be used for commercial purposes. To see the complete license contents, please visit http://creativecommons.org/licenses/by-nc/3.0/. 